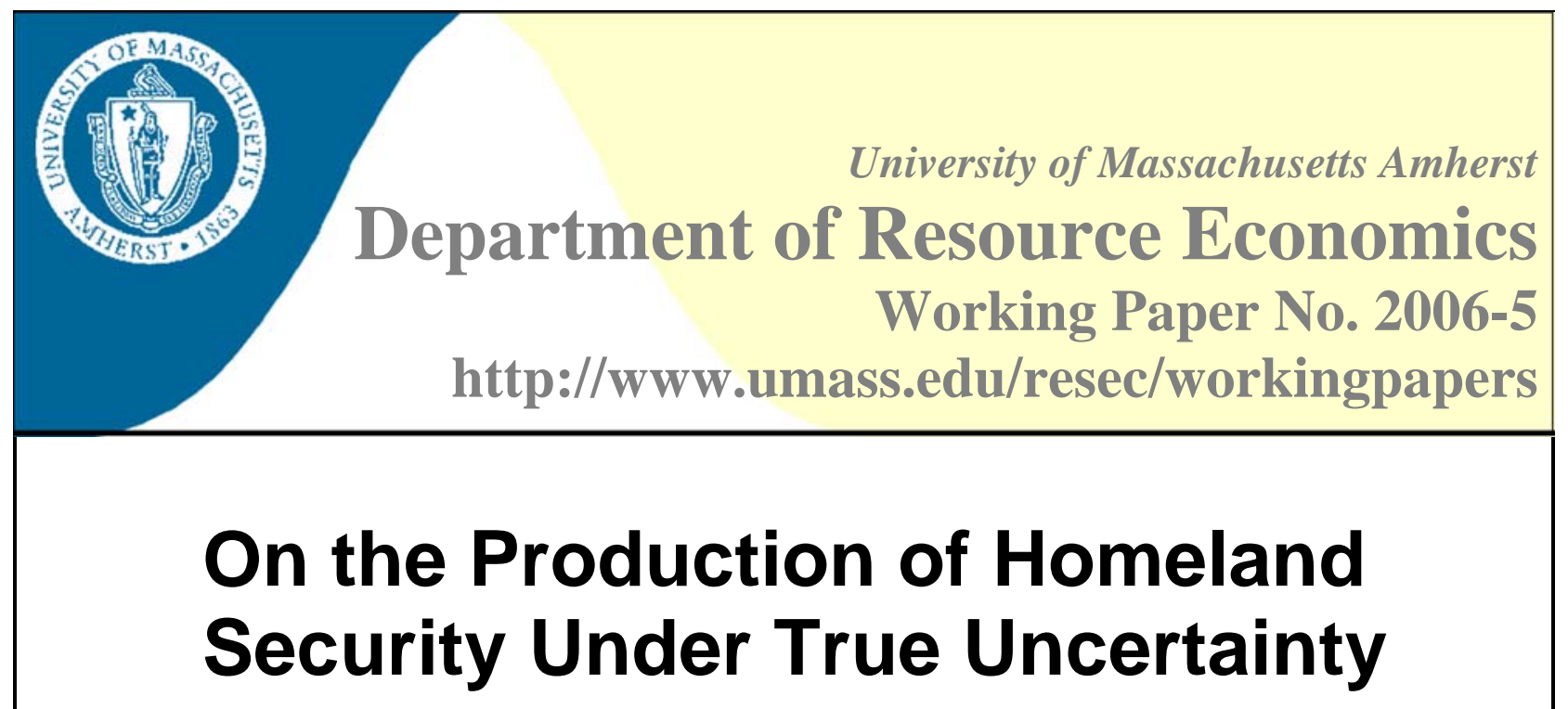

\author{
John K. Stranlund ${ }^{1}$ and Barry C. Field ${ }^{2}$
}

Abstract:

Homeland security against possible terrorist attacks involves making decisions under true uncertainty. Not only are we ignorant of the form, place, and time of potential terrorist attacks, we are also largely ignorant of the likelihood of these attacks. In this paper, we conceptualize homeland security under true uncertainty as society's immunity to unacceptable losses. We illustrate and analyze the consequences of this notion of security with a simple model of allocating a fixed budget for homeland security to defending the pathways through which a terrorist may launch an attack and to mitigating the damage from an attack that evades this defense. In this problem, immunity is the range of uncertainty about the likelihood of an attack within which the actual expected loss will not exceed some critical value. We analyze the allocation of a fixed homeland security budget to defensive and mitigative efforts to maximize immunity to alternative levels of expected loss. We show that the production of homeland security involves a fundamental trade-off between immunity and acceptable loss; that is, for fixed resources that are optimally allocated to defense and mitigation, increasing immunity requires accepting higher expected losses, and reducing acceptable expected losses requires lower immunity. Greater investments in homeland security allow society to increase its immunity to a particular expected loss, reduce the expected losses to which we are immune while holding the degree of immunity constant, or some combination of increased immunity to a lower critical expected loss.

Keywords: Homeland Security, Terrorism, True Uncertainty.

JEL Classification: D02, D81, H56

\footnotetext{
${ }^{1}$ John K Stranlund, Department of Resource Economics

University of Massachusetts, Stockbridge Hall, 80 Campus Center Way, Amherst, MA 01003

E: stranlund@resecon.umass.edu P: 413-545-6328 F: 413-545-5853

${ }^{2}$ Barry C. Field, Department of Resource Economics

University of Massachusetts, Stockbridge Hall, 80 Campus Center Way, Amherst, MA 01003

E: field@resecon.umass.edu P: 413-545-5709 F: 413-545-5853
} 
August 2006

\title{
On the Production of Homeland Security Under True Uncertainty
}

\author{
JOHN K. STRANLUND* \\ Department of Resource Economics \\ University of Massachusetts-Amherst \\ BARRY C. FIELD \\ Department of Resource Economics \\ University of Massachusetts-Amherst
}

Acknowledgements: Funding for this research was provided by the U. S. Department of Agriculture under USDA/ERS/PREISM Cooperative Agreement No. 43-3AEM-4-80115, and the Cooperative State Research Extension, Education Service, U. S. Department of Agriculture, Massachusetts Agricultural Experiment Station under Project No. MAS00861. The authors gratefully acknowledge helpful comments from L. Joe Moffitt and Yakov Ben-Haim.

\footnotetext{
${ }^{*}$ Correspondence to John K. Stranlund, Department of Resource Economics, 80 Campus Center Way, 214 Stockbridge Hall, University of Massachusetts-Amherst, Amherst, MA 01003, USA. Phone: (413)545-6328, Fax: (413)545-5853, E-mail: stranlund@resecon.umass.edu.
} 


\title{
On the Production of Homeland Security Under True Uncertainty
}

\begin{abstract}
Homeland security against possible terrorist attacks involves making decisions under true uncertainty. Not only are we ignorant of the form, place, and time of potential terrorist attacks, we are also largely ignorant of the likelihood of these attacks. In this paper, we conceptualize homeland security under true uncertainty as society's immunity to unacceptable losses. We illustrate and analyze the consequences of this notion of security with a simple model of allocating a fixed budget for homeland security to defending the pathways through which a terrorist may launch an attack and to mitigating the damage from an attack that evades this defense. In this problem, immunity is the range of uncertainty about the likelihood of an attack within which the actual expected loss will not exceed some critical value. We analyze the allocation of a fixed homeland security budget to defensive and mitigative efforts to maximize immunity to alternative levels of expected loss. We show that the production of homeland security involves a fundamental trade-off between immunity and acceptable loss; that is, for fixed resources that are optimally allocated to defense and mitigation, increasing immunity requires accepting higher expected losses, and reducing acceptable expected losses requires lower immunity. Greater investments in homeland security allow society to increase its immunity to a particular expected loss, reduce the expected losses to which we are immune while holding the degree of immunity constant, or some combination of increased immunity to a lower critical expected loss.
\end{abstract}

Keywords: Homeland Security, Terrorism, True Uncertainty.

JEL Classifications: D02, D81, H56

\section{Introduction}

There are essentially three ways to protect a population from terrorist attacks: (1) neutralizing terrorists before they can mount attacks, (2) stopping attacks after they have been started but before they are completed, and (3) taking steps to reduce the severity of successful attacks. There clearly exists an economic problem in deciding how to divide fixed resources among these different functions. The last two, defending pathways through which an attack may occur and mitigating the effects of a failure of this defense, generally correspond to the responsibilities of the U.S. Department of Homeland Security (DHS). A substantial part of its budget goes to a variety of efforts to intercept terrorist attacks before they can be consummated. Chief among these are the inspection programs put into place in the air, land, and sea entry ports of the country. Another portion of its budget goes to hardening potential targets and developing strategies to mitigate damage from attacks that elude detection. 
Given known, or confidently estimated probability density functions over the form, place, and time of potential terrorist attacks, one could cast the problem of investing in security against terrorist attacks in the familiar terms of risk analysis. ${ }^{1}$ For one example among several possibilities, one could model the choices of defense and mitigation to minimize the expected losses from terrorist attacks. In principle, probability distribution functions over terrorist attacks can be estimated from the frequency of past attacks. But while there has been significant work to develop and examine time series of terrorist events over reasonably long periods (Enders and Sandler, 2002; Mickolus et al., 1989 and 1993; Enders et al., 1992; O’Brien, 1996) it is not straightforward to turn frequencies of past attacks into current attack probabilities. Terrorists can choose innovative tactics, as happened on 9-11-01, in ways that are not readily predictable from past actions. Moreover, technological change (e.g., the internet), the rapid pace of globalization, and the ebb and flow of political movements produce novel opportunities that can only be roughly characterized by past attacks. Under contemporary circumstances, we are extremely pessimistic about our ability to estimate probability distribution functions over terrorist attacks with any degree of confidence. What this strongly suggests is that it may not be useful to think about homeland security against terrorism as decisions involving gambles with known probabilities. Instead, with respect to homeland security, we are truly in a world of Knightian uncertainty; that is, not only are we ignorant of the form, place, and time of potential terrorist attacks, we are also ignorant of the likelihood of these attacks. ${ }^{2}$ Thus, any useful characterization of the definition and social choice of homeland security must account for this uncertainty.

In this paper we propose that security under true uncertainty can be usefully thought of as the degree of immunity against unacceptable expected losses from terrorist attacks. Moreover, it is reasonable to assume that society's preference for security is monotonically increasing in the immunity to a particular loss, and decreasing in this loss while holding immunity constant. That is, we are more secure if we can achieve greater immunity to a particular critical loss, if we can reduce the critical loss without affecting the degree of immunity to this loss, or if we can achieve both increased immunity to lower loss.

\footnotetext{
${ }^{1}$ This approach has been taken by Kunreuther and Heal (2003), Heal and Kunreuther (2005), Keohane and Zeckhauser (2003), Lakdawalla and Zanjani (2005), Bueno de Mesquita (2005), and others.

${ }^{2}$ Knight was concerned "with situations which are far too unique, generally speaking, for any sort of statistical tabulation to have any value for guidance. The conception of an objectively measurable probability or chance is simply inapplicable." Knight referred to this as true or unmeasurable uncertainty (Knight, 1921, chapter 7).
} 
We illustrate and analyze the consequences of this notion of security with a simple model of allocating a fixed budget to homeland security for efforts to stop a terrorist attack and efforts to mitigate the damage from an attack that evades our defenses. Within this problem immunity is the range of uncertainty about the likelihood of an attack within which the actual expected loss will not exceed some critical value. With this definition of security, we analyze the allocation of a fixed homeland security budget to defensive and mitigative efforts to maximize security to a truly uncertain terrorist attack. We demonstrate that defense is a normal input into the production of homeland security, while mitigation is an inferior input. That is, as society increases its investment in homeland security, more of these resources should be devoted to defense and less to mitigation. Moreover, we show that the production of homeland security involves a fundamental trade-off between immunity and acceptable loss; that is, for fixed resources that are optimally allocated to defense and mitigation, increasing immunity to loss requires accepting higher expected losses, and reducing acceptable expected losses requires lower immunity. Increasing security in the sense of increasing immunity to a particular expected loss, reducing the critical expected loss for the same degree of immunity, or a combination of increased immunity to a lower critical expected loss, is achievable only with a greater investment in homeland security.

Several approaches have been developed to analyze decision making under true uncertainty. These approaches include application of the maximin, maximax, Laplace, and Hurwitz criteria (Render et al., 2003). While none of these criteria require knowledge of probability distributions for application, the first two represent polar extremes in terms of optimism and pessimism while the latter two require information similar to probabilities in order to be applied. Similarly, quantification of other notions related to uncertainty such as ignorance and surprise have also required the specification of functions confined to the unit interval (Katzner, 1998; Horan et al., 2002). Additionally, Kelsey (1993) has proposed a decision theory requiring a ranking of event probabilities rather than a specific probability distribution. None of these decision criteria under uncertainty have achieved the widespread application in economics afforded traditional risk criteria. More importantly for our purposes, none of these decision criteria provide a natural conceptualization of the definition and pursuit of security under true uncertainty. 
Our analysis is an application of Ben-Haim’s (2006) information-gap decision theory. ${ }^{3}$ The heart of Ben-Haim's approach is the pursuit of decisions that are robust in the sense that they maximize the range of uncertainty about model parameters within which the decision maker is certain to achieve a performance criterion. ${ }^{4}$ In our problem of homeland security, we are uncertain about the likelihood of a terrorist attack but we seek to maximize the range of this uncertainty over which the expected loss from an attack will not exceed some critical value. Thus, Ben-Haim’s approach provides a useful way to define homeland security as immunity to unacceptable losses and to analyze its production.

\section{Homeland Security Under True Uncertainty}

The provision of homeland security against terrorism is exceedingly complex, involving defending the literally thousands of avenues through which terrorists might conceivably attack and investing in at least as many methods by which the effects of a successful attack can be mitigated. Our purpose, however, is not to model homeland security in all its complexity, but rather to formalize a useful definition of security against uncertain terrorist attacks and to analyze certain characteristics of its production. To that end we examine a situation in which a terrorist attack may be launched with an unknown probability through a large number of potential pathways. The number of these pathways is large enough that defending all of them is prohibitively costly. Since not all pathways can be defended, there is some likelihood that a terrorist attack will be successful, hence society invests in efforts to mitigate the loss from a successful attack. Though highly stylized, our approach is applicable to any situation involving defending one's borders against an attack that occurs with unknown probability and mitigating the effects of a failure in this defense.

\footnotetext{
${ }^{3}$ Ben-Haim's decision theory has been applied to a wide variety of problems, including financial risk assessment (Ben-Haim 2005), search behavior in animal foraging models (Carmel and Ben-Haim 2005), policy decisions in marine reserve design (Halpern et al. 2006), natural resource conservation decisions (Moilanen et al. 2006), inspection decisions by port authorities to detect terrorist weapons (Moffitt et al. 2005a) and invasive species (Moffitt et al. 2005b), technological fault diagnosis (Pierce et al. 2006) and engineering model-testing (Vinot et al. 2005).

${ }^{4}$ This approach is related to recent attempts to develop robust monetary policy, where the source of uncertainty is unknown variation in the underlying monetary models that are used to derive policy rules. See the papers on robust decisions in Macroeconomic Dynamics, Vol. 6, No., 1, February 2002. Policy rules are evaluated using a reference model of the workings of the monetary system. Important parameters of the model are then perturbed, leading to variations in outcomes under the policy rule. The robustness of a rule is the maximum perturbation that can be allowed while keeping the outcome of the rule within specified bounds. By comparing alternative rules under this procedure one can identify the one with maximal robustness.
} 


\subsection{A model of homeland security}

Let $N$ denote the number of pathways through which an attack on a nation may occur. The probability of an attack that may occur through any one of these pathways is $p$. The uncertainty in this model is about $p$ - the probability that a terrorist has launched an attack is completely unknown. The pathways are identical in all regards, so a terrorist is indifferent about which pathway to attack. To defend against a potential attack, $n \leq N$ of the pathways are defended. Since they are homogeneous, defended pathways are chosen at random. An attack on a defended pathway will be thwarted. Thus, the probability of a successful attack is the probability that an attack has been launched times the probability that the weapon gets through the nation's defenses, $p(N-n) / N$.

A successful terrorist attack results in a certain loss $L .^{5}$ Mitigation efforts, $m$, reduce this potential loss, but at a decreasing rate. That is, the loss from a successful attack is $L(m)$, with $L^{\prime}(m)<0$ and $L^{\prime \prime}(m)>0$. Given the probability of an attack, $p$, the expected loss from an attack is $L(m) p(N-n) / N$. This value is unknown because the probability of an attack is completely unknown. We might, however, have complete confidence that the probability of an attack is no more than some value $p_{c} \leq 1$; that is, we may be uncertain of the true probability of an attack, but we are certain that it does not exceed $p_{c}$.

\subsection{The definition of homeland security under true uncertainty}

We are now ready to formalize a definition of homeland security as the degree of immunity against unacceptable loss. Let $\bar{L}$ be a critical value for the expected loss from a terrorist attack, and consider the problem of maximizing the range of the probability of an attack within which the expected loss from a possible attack does not exceed $\bar{L}$; that is,

$$
\max _{p \in\left[0, p_{c}\right]} L(m) p(N-n) / N \leq \bar{L}, n \in[0, N], m \geq 0
$$

\footnotetext{
${ }^{5}$ There are likely to be random elements of the damage caused by a successfully deployed weapon, and these elements may even be truly uncertain. We assume that this loss is known with certainty in order to focus on one uncertain element, that is, on the probability that an attack has been launched.
} 
Given defense and mitigation efforts, $L(m) p(N-n) / N$, is increasing in $p$. Therefore, the solution to [1] is to set $L(m) p(N-n) / N=\bar{L}$ and solve for $p$ to obtain

$$
p\left(n, m ; \bar{L}, p_{c}\right)=\frac{\bar{L} N}{L(m)(N-n)}, p \in\left[0, p_{c}\right], n \in[0, N], m \geq 0
$$

The function $p\left(n, m ; \bar{L}, p_{c}\right)$ characterizes security against an uncertain attack in the sense of immunity to potential loss. Specifically, given defense and mitigation efforts, $p\left(n, m ; \bar{L}, p_{c}\right)$ is the maximum probability of an attack for which we are certain that the expected loss does not exceed the critical value $\bar{L}$. In other words, society is immune to expected loss $\bar{L}$ as long as the actual probability of an attack does not exceed $p\left(n, m ; \bar{L}, p_{c}\right)$.

\subsection{The technology of security}

Equation [2] describes the 'technology' of homeland security under true uncertainty. It characterizes society's opportunities for taking actions (defense and mitigation) to achieve alternative degrees of immunity to alternative levels of expected loss. In the next section, we will characterize the 'production' of homeland security as the allocation of defense and mitigation efforts that maximizes [2], given the resources devoted to homeland security. Toward this end we first need to describe how $p\left(n, m ; \bar{L}, p_{c}\right)$ varies with the number of defended pathways and efforts to mitigate the effects of a successful attack. The following proposition, which is proved in the appendix, provides this description:

Proposition 1: For $p\left(n, m ; \bar{L}, p_{c}\right)<p_{c}, n \in(0, N)$, and $m>0, p\left(n, m ; \bar{L}, p_{c}\right)$ has the following characteristics:

i) $p_{n}>0, p_{m}>0$, and $p_{n m}>0$;

ii) $p_{n n}>0$, while the sign of $p_{m m}$ is indeterminate;

iii) $p\left(n, m ; \bar{L}, p_{c}\right)$ is strictly quasi-concave in $(n, m)$ if and only if $p_{m m}<0 . p\left(n, m ; \bar{L}, p_{c}\right)$ is strictly quasi-convex if and only if $p_{m m}>0$. 
Part $i)$ of the proposition indicates that $p\left(n, m ; \bar{L}, p_{c}\right)$ is montonically increasing in $n$ and $m$ so that increasing the number of defended pathways and increasing mitigation efforts both increase society's immunity to the critical expected loss $\bar{L}$. Moreover, $p_{n m}>0$ implies that defense and mitigation are complements in the sense that increased mitigation increases the marginal productivity of defense, and vice-versa. Part ii) of the proposition reveals that the marginal productivity of defense increases as more pathways are defended $\left(p_{n n}>0\right)$. On the other hand, it is unclear how increased mitigation affects the marginal productivity of mitigation. If $p_{m m}<0$, then increasing mitigation increases society's immunity to a critical expected loss, but at a decreasing rate. On the other hand, if $p_{m m}>0$ then the marginal productivity of mitigation increases with greater mitigation effort. Part iii) of the proposition reveals that whether the marginal productivity of mitigation is increasing or decreasing determines whether

$p\left(n, m ; \bar{L}, p_{c}\right)$ is quasi-concave or quasi-convex. This distinction has implications for the optimal allocation of homeland security resources to defense and mitigation, which we explore next.

\section{The Production of Homeland Security}

Obviously, levels of homeland security depend on the resources devoted to it. Moreover, given the resources devoted to security, maximal security is attained by the efficient allocation of these resources to defense and mitigation efforts. Let $R$ denote the monetary resources devoted to homeland security, and let $w_{n}$ and $w_{m}$ denote the unit costs of defense and mitigation efforts, respectively. Then, the efficient allocation of resources to defense and mitigation to maximize immunity to a particular expected loss is the solution to:

$$
\begin{array}{ll}
\max _{n, m} & p\left(n, m, \bar{L}, p_{c}\right)=\bar{L} N /(L(m)(N-n)) \\
\text { s.t. } & R \geq w_{n} n+w_{m} m \\
& p\left(n, m ; \bar{L}, p_{c}\right) \leq p_{c} \\
& n \in[0, N], m \geq 0 .
\end{array}
$$


At the outset this optimization problem can be simplified a bit. First, the resource constraint always binds. The monotonicity of $p\left(n, m ; \bar{L}, p_{c}\right)$ in $n$ and $m$ implies that if $R>w_{n} n+w_{m} m$, then $p\left(n, m ; \bar{L}, p_{c}\right)=p_{c}$. But then security can be increased by decreasing $\bar{L}$ while increasing $n$ and/or $m$ until the budget is exhausted.

There is a possible solution to [3] than involves defending all pathways against possible attack. Since $p\left(n, m, \bar{L}, p_{c}\right)=\bar{L} N /(L(m)(N-n))$, the constraint $p\left(n, m ; \bar{L}, p_{c}\right) \leq p_{c}$ can be written as $\bar{L} \leq p_{c} L(m)(N-n) / N$. Setting $n=N$ so that all pathways are defended allows us to be perfectly immune to any expected loss; that is, we can achieve $p\left(n, m ; \bar{L}, p_{c}\right)=p_{c}$ for $\bar{L}=0$. It seems to us, though, that defending all potential targets of a terrorist attack is likely to be prohibitively expensive. Therefore, from here on, we only examine solutions to [3] that involve $n$ $<N$.

Moreover, let us assume that $p\left(n, m ; \bar{L}, p_{c}\right)$ is strictly quasi-concave in $(n, m)$ so that we can focus on solutions to [3] that involve $n>0$ and $m>0 .{ }^{6}$ (We will briefly consider situations involving $n=0$ or $m=0$ later). Then the choices of defense and mitigation solve $\max _{n, m} p\left(n, m, \bar{L}, p_{c}\right)$ subject to $R-w_{n} n-w_{m} m=0$. Let $\mathcal{L}$ denote the Lagrange equation for this problem and let $\lambda$ denote the multiplier for the budget constraint. Under our assumptions the following first-order conditions are both necessary and sufficient to solve [3]:

$$
\begin{aligned}
& \mathcal{L}_{n}=\frac{\bar{L} N}{L(m)(N-n)^{2}}-\lambda w_{n}=0 ; \\
& \mathcal{L}_{m}=\frac{-\bar{L} N L^{\prime}(m)}{[L(m)]^{2}(N-n)}-\lambda w_{m}=0 ; \\
& \mathcal{L}_{\lambda}=R-w_{n} n-w_{m} m=0 .
\end{aligned}
$$

\footnotetext{
${ }^{6}$ Quasi-concavity or quasi-convexity determines the curvature of the level curves of $p\left(n, m ; \bar{L}, p_{c}\right)$. If $p\left(n, m ; \bar{L}, p_{c}\right)$ is strictly quasi-convex, then its level curves are strictly concave. In these cases, the optimal choices of defense and mitigation will certainly be a corner solution.
} 
Denote the solution to [4]-[6] as $\left(n^{*}, m^{*}, \lambda^{*}\right)$. The following proposition characterizes how security-maximizing choices of defense and mitigation depend on the critical loss $\bar{L}$ and the resources devoted to security $R$. It is proved in the appendix.

Proposition 2: Provided that $p\left(n, m ; \bar{L}, p_{c}\right)$ is strictly quasi-concave and $n^{*}>0$ and $m^{*}>0$ :

i) $n^{*}$ and $m^{*}$ are independent of $\bar{L}$;

ii) $n^{*}$ is increasing in $R$ while $m^{*}$ is decreasing in $R$.

Part $i$ ) indicates that optimal defense and mitigation are independent of the critical expected loss. This is true because $p\left(n, m ; \bar{L}, p_{c}\right)$ is a linear function of $\bar{L}$.

Part ii) of the proposition reveals that the number of defended pathways is a normal input in the production of security, while mitigation is an inferior input. Thus, increased resources allocated to homeland security should be devoted to defense, while at the same time decreasing mitigation efforts. The intuition behind this result is straightforward. Clearly, since the marginal productivity of defense is increasing in higher levels of defense (i.e., $p_{n n}>0$ ), society should exploit this by allocating at least a part of an increase in security resources devoted to increased defense. However, doing so must be accompanied by a decrease in mitigation efforts. To understand why this must be the case, note that the first-order conditions [4] and [5] imply that defense and mitigation be chosen so that the ratio of the marginal products of these efforts in producing security is equal to the ratio of the prices of these efforts. That is, [4] and [5] can be combined to yield $p_{n} / p_{m}=w_{n} / w_{m}$. Allocating at least a part of an increase in security resources to additional defense increases $p_{n}$, because $p_{n n}>0$. To maintain $p_{n} / p_{m}=w_{n} / w_{m}$, then, mitigation must change to increase $p_{m}$ by the same amount as the increase in $p_{n}$. Since the marginal productivity of mitigation is decreasing in this effort $\left(p_{m m}<0\right)$, increasing $p_{m}$ is accomplished by reducing mitigation. 
We will not be analyzing the effects of changes in the costs of defense and mitigation on the optimal solution. ${ }^{7}$ Therefore, Proposition 3 allows us to simply write the optimal values for defense and mitigation in terms of the resources devoted to homeland security; that is $n^{*}=n^{*}(R)$ and $m^{*}=m^{*}(R)$. Then, maximal immunity to expected loss $\bar{L}$ is

$$
p\left(\bar{L}, R, p_{c}\right)=p\left(n^{*}, m^{*} ; \bar{L}, p_{c}\right)=\frac{\bar{L} N}{L\left(m^{*}(R)\right)\left(N-n^{*}(R)\right)} \leq p_{c} .
$$

Clearly, with respect to $\bar{L}, \bar{L} N /\left(L\left(m^{*}\right)\left(N-n^{*}\right)\right)$ has a zero intercept and is linearly increasing (this last follows because $n^{*}$ and $m^{*}$ are independent of $\bar{L}$ ).

Figure 1 is a graph of $\bar{L} N /\left(L\left(m^{*}\right)\left(N-n^{*}\right)\right)$ that we use to further refine the production function for security. As seen in the graph, for sufficiently high values of $\bar{L}$, $\bar{L} N /\left(L\left(m^{*}\right)\left(N-n^{*}\right)\right)$ exceeds $p_{c}$. An example of such an outcome is $\left(p_{+}, \bar{L}_{+}\right)$. Clearly, since $p_{+}>p_{c}$, immunity to $\bar{L}_{+}$exceeds the maximum probability of a terrorist attack. Then, one may be tempted to simply say that maximal security is given by the point $\left(p_{c}, \bar{L}_{+}\right)$in the graph. Doing so suggests that optimal security $p\left(\bar{L}, R, p_{c}\right)$ increases up to $p_{c}$ and then is constant at this level for higher levels of critical loss $\bar{L}$. However, a point like $\left(p_{c}, \bar{L}_{+}\right)$cannot represent the maximal security attainable with resources $R$, because such an outcome implies that society is willing to accept higher expected losses than it needs to. Reducing the expected critical loss value from $\bar{L}_{+}$to $\bar{L}_{k}$ while holding immunity to $p_{c}$ represents greater security without additional costs. Therefore, the optimal production of security is defined only over expected critical losses between zero and $\bar{L}_{k}$, inclusive. Thus, the following proposition completely characterizes the optimal production of homeland security.

\footnotetext{
${ }^{7}$ As one would guess, both defense and mitigation are decreasing in their respective unit costs. The cross-cost effects - the effect on defense of an increase in the unit cost of mitigation and the effect on mitigation of an increase in the unit cost of defense-are ambiguous.
} 
Proposition 3: Given resources $R$ devoted to homeland security, its optimal production is:

$$
p\left(\bar{L}, R, p_{c}\right)=\frac{\bar{L} N}{L\left(m^{*}(R)\right)\left(N-n^{*}(R)\right)}, \quad \bar{L} \in\left[0, \bar{L}_{k}\left(R, p_{c}\right)\right],
$$

where

$$
\bar{L}_{k}\left(R, p_{c}\right)=\left\{\bar{L} \mid \frac{N \bar{L}}{L\left(m^{*}(R)\right)\left(N-n^{*}(R)\right)}=p_{c}\right\}=p_{c} L\left(m^{*}(R)\right)\left(N-n^{*}(R)\right) / N .
$$

Our final proposition provides the fundamental characteristics of the optimal production of homeland security. It is proved in the appendix.

Proposition 4: i) $p\left(\bar{L}, R, p_{c}\right)=0$ for $\bar{L}=0$;

ii) $p\left(\bar{L}, R, p_{c}\right)$ is linearly increasing in $\bar{L}$ up to $p_{c}$;

iii) $p\left(\bar{L}, R, p_{c}\right)$ is increasing and strictly convex in $R$, for $\bar{L}<\bar{L}_{k}\left(R, p_{c}\right)$;

iv) $\partial^{2} p\left(\bar{L}, R, p_{c}\right) / \partial R \partial \bar{L}>0$, for $\bar{L}<\bar{L}_{k}\left(R, p_{c}\right)$;

Part $i$ ) of the proposition indicates zero immunity against zero expected loss; that is, society has no confidence that the expected loss from a terrorist attack is zero. Part ii) indicates a fundamental tradeoff between immunity and expected loss. Given resources devoted to homeland security that are optimally allocated to defense and mitigation, increased immunity against unacceptable expected losses is attained only by tolerating higher expected losses. However, parts iii) and iv) indicate that devoting more resources to homeland security allows the attainment of greater immunity to a particular expected loss, lower critical expected loss for the same degree of immunity, or some combination of greater immunity to lower critical expected loss. Moreover, given a critical expected loss $\bar{L} \in\left(0, \bar{L}_{k}\left(R, p_{c}\right)\right)$, immunity to this loss increases at an increasing rate with greater resources devoted to homeland security. 
Figure 2 is a graph of $p\left(\bar{L}, R, p_{c}\right)$ for two resource levels, $R_{0}<R_{1}$, which we can use to illustrate the main results of this paper. In the graph, $\bar{L}_{0}=\bar{L}_{k}\left(R_{0}, p_{c}\right)$ and $\bar{L}_{1}=\bar{L}_{k}\left(R_{1}, p_{c}\right)$ as defined by [8].

Homeland security under true uncertainty about the probability of a terrorist attack is the degree of immunity to unacceptable expected losses. More rigorously, a point like $\left(p_{A}, \bar{L}_{A}\right)$ in Figure 2 indicates the maximum probability of a terrorist attack, $p_{A}$, up to which we are certain that the expected loss from such an attack does not exceed $\bar{L}_{A}$. Suppose that society devotes $R_{0}$ resources to defending against a terrorist attack and mitigating the effects of a successful attack, but achieves only $\left(p_{A}, \bar{L}_{A}\right)$. Clearly, the allocation of $R_{0}$ to defense and mitigation is inefficient because greater security can be achieved with these same resources. Combinations of immunity and critical expected loss on the $a b$ segment of $p\left(\bar{L}, R_{0}, p_{c}\right)$ represent points of greater security than $\left(p_{A}, \bar{L}_{A}\right)$, because they involve greater immunity to expected loss $\bar{L}_{A}$, reduced expected loss while keeping immunity to that loss constant at $p_{A}$, or some combination of greater immunity to lower acceptable expected loss like point $\left(p_{B}, \bar{L}_{B}\right)$.

Given that society efficiently allocates $R_{0}$ to defense and mitigation, changes in security involve a fundamental tradeoff between immunity and loss. Achieving greater immunity requires tolerating a higher potential expected loss, and vice versa. (Indeed, reducing the allowable loss to zero requires reducing immunity to zero). Achieving greater security requires a greater investment in homeland security. This is illustrated in Figure 2 where an increase in this investment from $R_{0}$ to $R_{1}$, allows the achievement of points of greater security than $\left(p_{B}, \bar{L}_{B}\right)$ on the $c d$ segment of $p\left(\bar{L}, R_{1}, p_{c}\right)$. Interestingly, since defense is normal input into the production of security and mitigation is an inferior input (Proposition 2, part ii)), the increase in security resources from $R_{0}$ to $R_{1}$ calls for allocating more of these resources to defense and less to mitigation.

To complete the analysis let us very briefly examine how possible corner solutions for the choice of defense or mitigation affect our main results. These situations may occur when $p\left(n, m ; \bar{L}, p_{c}\right)$ is quasi-convex in $(n, m)$. Or, given that we know that defense is a normal input into the production of security while mitigation in an inferior input, very high levels of homeland 
security resources may call for devoting all of these resources to defense and none to mitigation. Even in these corner-solution cases, $p\left(\bar{L}, R, p_{c}\right)$ retains its essential characteristics provided in Proposition 4. If $n^{*}=0$, all security resources are allocated to mitigation so that $m^{*}=R / w_{m}$. Then, $p\left(\bar{L}, R, p_{c}\right)=\bar{L} / L\left(R / w_{m}\right)$. As in Proposition $4, p\left(\bar{L}, R, p_{c}\right)=0$ for $\bar{L}=0$ and is linearly increasing in $\bar{L}$. Moreover, it is easy to show $\partial p\left(\bar{L}, R, p_{c}\right) / \partial R=-\bar{L} N L^{\prime}\left(m^{*}\right) / w_{m}\left[L\left(m^{*}\right)\right]^{2}>0$, indicating that $p\left(\bar{L}, R, p_{c}\right)$ is increasing in $R$. On the other hand, if $m^{*}=0$, then $n^{*}=R / w_{n}$ and $p\left(\bar{L}, R, p_{c}\right)=\bar{L} N / L(0)\left(N-R / w_{n}\right)$. It is straightforward to show that in these cases, $p\left(\bar{L}, R, p_{c}\right)$ retains the same basic characteristics. The upshot then is that corner choices of defense and mitigation do not change the fundamental structure of the production of homeland security as characterized by Proposition 4.

\section{Concluding Remarks: The Social Choice of Homeland Security}

We have examined the definition and production of homeland security under true uncertainty about terrorist attacks. We have argued that the degree of immunity to unacceptable expected losses from terrorist attacks is a useful way to conceptualize security under true uncertainty. We have illustrated this concept of security with a model of allocating a fixed budget for homeland security to defending the pathways through which a terrorist may launch an attack and to efforts to mitigate the damage from an attack that evades this defense. Immunity to unacceptable losses in this problem is the range of uncertainty about the likelihood of an attack within which the actual expected loss will not exceed some critical value. Homeland security resources are optimally allocated to defense and mitigation to maximize immunity to alternative levels of expected loss. Our most important result is that the production of homeland security involves a fundamental trade-off between immunity and acceptable loss; that is, for fixed resources that are optimally allocated to defense and mitigation, increasing immunity to loss requires accepting higher expected losses, and reducing acceptable expected losses requires lower immunity. Greater investments in homeland security allow society to increase security by increasing immunity to some critical expected loss, reducing the expected loss we are willing to tolerate while holding immunity to this loss constant, or some combination of increased immunity to a lower critical expected loss. 
Although we have shown a useful way to think about the problem of homeland security and have analyzed characteristics of its production, we have said little about society's preference and choice of security. Indeed, the variables that are important for defining and producing security—degree of immunity, critical expected loss, and the resources devoted to homeland security - are all matters of social choice. We have assumed that society prefers greater immunity to lower expected loss, but we have shown that achieving both with fixed resources is not possible. Therefore, a fuller description of the relative values that society places on immunity and critical expected loss is necessary to analyze the social choice over these elements of homeland security. 


\section{Appendix}

Proof of Proposition 1: For parts i) and ii) use [2] to calculate

$$
\begin{aligned}
& p_{n}=\frac{\bar{L} N}{L(m)(N-n)^{2}}>0 ; p_{m}=\frac{-\bar{L} N L^{\prime}(m)}{[L(m)]^{2}(N-n)}>0 ; \\
& p_{n n}=\frac{2 \bar{L} N}{L(m)(N-n)^{3}}>0 ; p_{n m}=\frac{-\bar{L} N L^{\prime}(m)}{[L(m)]^{2}(N-n)^{2}}>0 ; \\
& p_{m m}=\frac{\bar{L} N\left\{2\left[L^{\prime}(m)\right]^{2}-L^{\prime \prime}(m) L(m)\right\}}{[L(m)]^{3}(N-n)^{3}} .
\end{aligned}
$$

Note that the sign of $p_{m m}$ is equal to the sign of $2\left[L^{\prime}(m)\right]^{2}-L^{\prime \prime}(m) L(m)$, and hence is indeterminate. For part iii), strict quasi-concavity requires

$$
2 p_{m} p_{n} p_{n m}-p_{n n}\left(p_{m}\right)^{2}-p_{m m}\left(p_{n}\right)^{2}>0
$$

Use the calculations above to show that $2 p_{m} p_{n} p_{n m}-p_{n n}\left(p_{m}\right)^{2}=0$. Therefore, $p\left(n, m ; \bar{L}, p_{c}\right)$ is strictly quasi-concave in $(n, m)$ if and only if $-p_{m m}\left(p_{n}\right)^{2}>0$, which requires $p_{m m}<0$. Clearly, if $p_{m m}>0$, then $p\left(n, m ; \bar{L}, p_{c}\right)$ is strictly quasi-convex, and $p\left(n, m ; \bar{L}, p_{c}\right)$ is strictly quasi-convex only if $p_{m m}>0$. QED.

Proof of Proposition 2: For part i), set [4] and [5] equal to each other and simplify the result to obtain

$$
w_{m} L(m)+w_{n} L^{\prime}(m)(N-n)=0 .
$$

This equation and the resource constraint, $R-w_{n} n-w_{m} m=0$, determine the optimal values for $n$ and $m$. Note that $\bar{L}$ does not enter either of these equations, implying that the optimal values of $n$ and $m$ are independent of this critical loss.

To prove part ii), note that the Hessian matrix associated with [4], [5], and [6] is 


$$
H=\left[\begin{array}{ccc}
p_{n n} & p_{n m} & -w_{n} \\
p_{m n} & p_{m m} & -w_{m} \\
-w_{n} & -w_{m} & 0
\end{array}\right] .
$$

The second order condition for an optimum is that the determinant of this matrix be strictly positive; that is $|H|>0$. The comparative statics with respect to the budget are the solutions to the system of equations

$$
H\left[\begin{array}{c}
n_{R}^{*} \\
m_{R}^{*} \\
\lambda_{R}^{*}
\end{array}\right]=\left[\begin{array}{r}
0 \\
0 \\
-1
\end{array}\right] .
$$

From [A.2] obtain $n_{R}^{*}=\left(p_{n m} w_{m}-p_{m m} w_{n}\right) /|H|>0$, The sign of $n_{R}^{*}$ follows because $p_{n m}>0$ from part ii) of Proposition 1 and $p_{m m}<0$, which is required if $p\left(n, m ; \bar{L}, p_{c}\right)$ is strictly quasiconcave.

From [A.2] obtain $m_{R}^{*}=\left(p_{n m} w_{n}-p_{n n} w_{m}\right) /|H|$. Calculate

$$
p_{n m} w_{n}-p_{n n} w_{m}=\frac{\bar{L} N\left[-w_{n} L^{\prime}(m)(N-n)-2 w_{m} L(m)\right]}{[L(m)]^{2}(N-n)^{3}} .
$$

From [A.1], $-w_{n} L^{\prime}(m)(N-n)=w_{m} L(m)$. Substituting this into $p_{n m} w_{n}-p_{n n} w_{m}$ reveals that $p_{n m} w_{n}-p_{n n} w_{m}<0$. Consequently, $m_{R}^{*}<0$. The proof is complete. QED.

Proof of Proposition 4: Part i) follows from [7] and the fact that $n^{*}$ and $m^{*}$ are independent of $\bar{L}$ (Proposition 2). For part ii), use the envelope theorem to obtain $\partial p\left(\bar{L}, R, p_{c}\right) / \partial R=\lambda^{*}$. If the first-order conditions [4] and [5] hold, then $\lambda^{*}>0$ and $\partial p\left(\bar{L}, R, p_{c}\right) / \partial R>0$. Note that $\partial^{2} p\left(\bar{L}, R, p_{c}\right) / \partial R^{2}=\lambda_{R}^{*}$. From the first order conditions [4], [5], and [6], and [A.2] obtain $\left.\lambda_{R}^{*}=\left[\left(p_{n m}\right)^{2}-p_{n n} p_{m m}\right)\right] /|H|>0$. The sign of $\lambda_{R}^{*}$ follows because $p_{n n}>0$ from part $\left.i i\right)$ of 
Proposition 1 and $p_{m m}<0$, which is required for $p\left(n, m ; \bar{L}, p_{c}\right)$ to be strictly quasi-concave. Finally, to prove part iv) of the proposition note that $\partial^{2} p\left(\bar{L}, R, p_{c}\right) / \partial R \partial \bar{L}=\lambda_{\bar{L}}^{*}$. From the firstorder condition [4], $\lambda^{*}=\bar{L} N / w_{n} L\left(m^{*}\right)\left(N-n^{*}\right)^{2}$. Since $n^{*}$ and $m^{*}$ are independent of $\bar{L}$, $\lambda_{\bar{L}}^{*}=N / w_{n} L\left(m^{*}\right)\left(N-n^{*}\right)^{2}>0$. This completes the proof. QED. 


\section{References}

Ben-Haim, Yakov. 2006. Info-Gap Decision Theory: Decisions Under Severe Uncertainty. Second Edition, Academic Press.

Ben-Haim, Yakov. 2005. "Value at Risk with Info-Gap Uncertainty.” Journal of Risk Finance 6(5), 388-403.

Bueno de Mesquita, Ethan. 2005. "The Terrorist Endgame, A Model with Moral Hazard and Learning.” Journal of Conflict Resolution 49(2), 237-258.

Carmel, Yohay and Yakov Ben-Haim. 2005. "Info-Gap Robust-Satisficing Model of Foraging Behavior: Do Foragers Optimize or Satisfice?” American Naturalist 166(5), 633-641.

Enders, W., and T. Sandler. 2002. "Patterns of Transnational Terrorism, 1970-99: Alternative Times Series Estimates.” International Studies Quarterly 46(2), 145-165.

Enders, W.; G. F. Parise and T. Sandler. 1992. "Time Series Analysis of Transnational Terrorism: Trends and Analysis.” Defence Economics 3(4), 305-320.

Halpern, Benjamin S.; Helen M. Regan, Hugh P. Possingham and Michael A. McCarthy. 2006. “Accounting for Uncertainty in Marine Reserve Design.” Ecology Letters 9, 2-11.

Heal, Geoffrey and Howard Kunreuther. 2005. “IDS Models of Airline Security.” Journal of Conflict Resolution 49(2), 201-217.

Horan, R. D., C. Perrings, F. Lupi, and E.H. Bulte. 2002. "Biological Pollution Prevention Strategies Under Ignorance: The Case of Invasive Species.” American Journal of Agricultural Economics 84(5), 1303-1310.

Katzner, D. W. Time, Ignorance, and Uncertainty in Economic Models. The University of Michigan Press. Ann Arbor, Michigan. 1998.

Kelsey, D. 1993. “Choice Under Partial Uncertainty.” International Economic Review 34(2), 297-308.

Keohane, Nathaniel O. and Richard J. Zeckhauser, 2003. “The Ecology of Terror Defense.” Journal of Risk and Uncertainty 26(2/3), 201-229.

Knight, Frank H. 1921. Risk, Uncertainty, and Profit. Houghton Mifflin Co. Re-issued by University of Chicago Press.

Kunreuther, Howard and Geoffrey Heal. 2003. “Interdependent Security.” Journal of Risk and Uncertainty 26(2/3), 231-249.

Lakdawalla, Darius and George Zanjani. 2005. "Insurance, Self-Protection, and the Economics of Terrorism.” Journal of Public Economics 89, 1891-1905.

Moilanen, Atte and Brendan A. Wintle. 2006. "Uncertainty Analysis Favours Selection of Spatially Aggregated Reserve Structures.” Biological Conservation 129(3), 427-434.

Mickolus, E. F.; T. Sandler, J. M. Murdock and P. Fleming. 1989. International Terrorism: Attributes of Terrorist Events, 1978-1987. (ITERATE 3). Dunn Loring, VA. Vinyard Software. 
Mickolus, E. F.; T. Sandler, J.M. Murdock, and P. Fleming. 1993. International Terrorism: Attributes of Terrorist Events, 1988-1991. (ITERATE 4). Dunn Loring, VA. Vinyard Software.

Moffitt, L. Joe; John K. Stranlund and Barry C. Field. 2005a. "Inspections to Avert Terrorism: Robustness under Severe Uncertainty.” Journal of Homeland Security and Emergency Management 2(3), Article 3. http://www.bepress.com/jhsem/vol2/iss3/3.

Moffitt L. Joe, John K. Stranlund, Barry C. Field, and Craig D. Osteen. 2005b. "Robust Inspection for Invasive Species with a Limited Budget." Forthcoming in The Economics of Plant Health. Alfons Oude Lansink (ed.). Springer.

O’Brien, S. P. 1996. "Foreign Policy Crisis and the Resort to Terrorism: A Time Series Analysis of Conflict Linkages.” Journal of Conflict Resolution 40(2), 320-335.

Pierce, S.G.; K. Worden and G. Manson. 2006. "A Novel Information-Gap Technique to Assess Reliability of Neural Network-Based Damage Detection.” Journal of Sound and Vibration 293(1-2), 96-111.

Render, B.; R. M. Stair Jr. and M. E. Hanna. 2006. Quantitative Analysis for Management. Ninth edition. Prentice Hall. Englewood Cliffs, NJ.

Vinot, P.; S. Cogan and V. Cipolla. 2005. “A Robust Model-Based Test Planning Procedure.” Journal of Sound and Vibration 288(3), 571-585. 


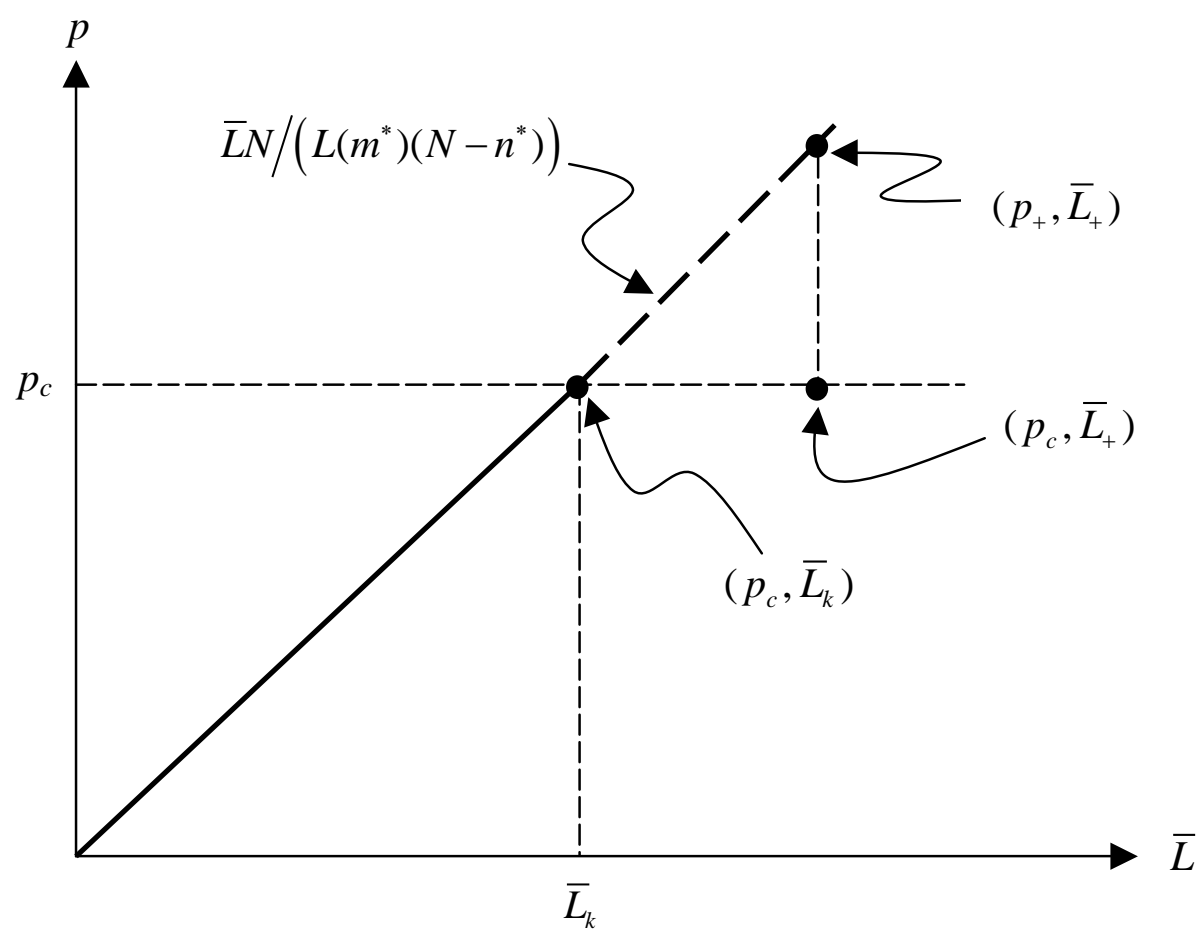

Figure 1: Refining the Production Function for Homeland Security. 


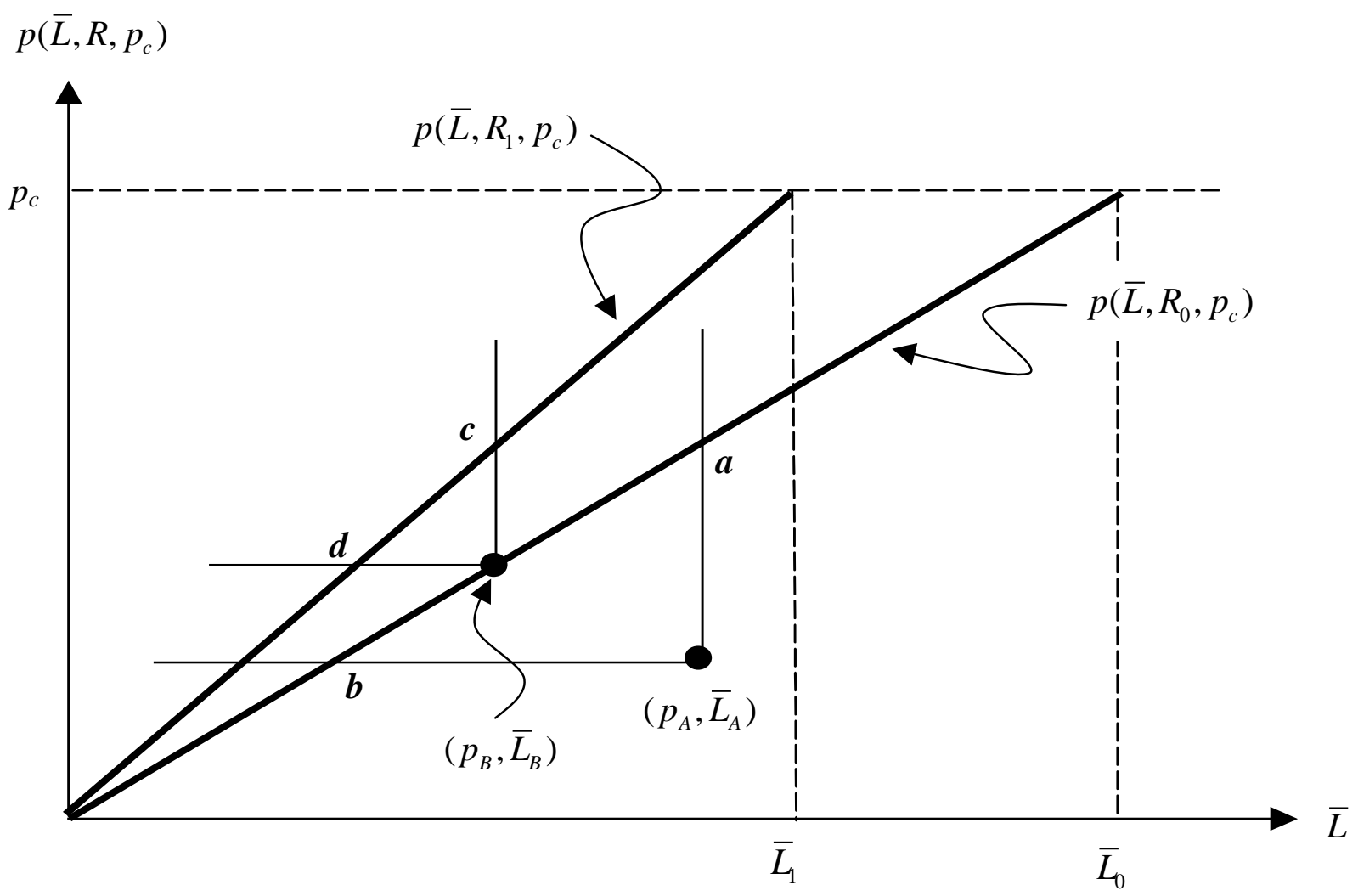

Figure 2: The Production of Homeland Security. 